\title{
Análise comparativa do efeito redutor de colesterol do extrato de semente e casca de uva em ratos Wistar
}

\section{Comparative analysis of reducing seed extract of cholesterol and effect bark uva in rats Wistar}

\author{
Thárcia Kiara Beserra de Oliveira \\ Doutoranda em Engenharia Agrícola, Universidade Federal de Campina Grande (UFCG) Campina Grande (PB). E-mail: tharcia_kiara@hotmail.com \\ Francisco de Assis Cardoso Almeida \\ Doutor em Engenharia Agrícola, (UFCG).E-mail: almeida.diassis@gmail.com \\ Flávio Paccelli Silva Sento Sé \\ Graduandos em Medicina na Faculdade de Ciências Médicas de Campina Grande. E-mail: paccelli_uni@ hotmail.com \\ Huggo Luãn Barros Medeiros \\ Graduandos em Medicina na Faculdade de Ciências Médicas de Campina Grande. E-mail: paccelli_uni@hotmail.com \\ Marina Suênia de Araújo Vilar \\ Doutora em Desenvolvimento e Inovação Tecnológica em Medicamentos - UFRN. E-mail: peritaquimica@yahoo.com.br \\ Inácia do Santos Moreira \\ Doutoranda em Engenharia Agrícola, Universidade Federal de Campina Grande (UFCG) Campina Grande (PB). E-mail: ceua@cesed.br
}

\begin{abstract}
Resumo: Os flavonóides são compostos fenólicos presentes na maioria das plantas. Têm um importante papel na prevenção e tratamento da aterosclerose, pois, atuam como agentes antiaterogênicos e hipocolesterolêmicos. Objetivouse nessa pesquisa estudar o efeito redutor de colesterol do extrato de semente e de casca de uva (Vitis vinifera) em ratos Wistar submetidos a uma dieta hipercalórica (ração acrescida de $10 \%$ de óleo vegetal) no período de 20 semanas, a partir da $4^{a}$ semana de vida. A amostra, composta por 30 espécimes e distribuída entre o Grupo Controle - A (com apenas dieta hipercalórica, $\mathrm{n}=10$ ) o Grupo Experimental Casca de Uva - B (dieta hipercalórica + extrato de casca de uva, $n=10$ ) e Grupo Experimental Semente de Uva - C (dieta hipercalórica + de extrato de semente de uva, n=10). Os resultados mostraram em média, uma redução dos níveis séricos de colesterol total de $14 \%$ e de $17,65 \%$ em relação ao grupo controle, respectivamente para os animais com dieta a base de casca e de semente de uva. Não houve diferença estatisticamente significativa na redução dos valores de colesterol entre os animais dos dois grupos, não sendo possível assim determinar qual componente da uva representa a melhor alternativa na prevenção e controle da hipercolesterolemia.
\end{abstract}

Palavras-chave: Dieta Hiperlipídica; Flavonóides; Hipercolesterolemia

\begin{abstract}
Flavonoids are phenolic compounds present in most plants. They play an important role in the prevention and treatment of atherosclerosis, as hypocholesterolemic and antiatherogenic act as agents. This research aimed to study and compare the cholesterol-lowering effect of seed extract and grape skin (Vitis vinifera) in rats subjected to a high calorie diet (increased ration of $10 \%$ vegetable oil) in the 20 -week period, from the 4 th week of life. The sample consisted of 30 specimens and distributed between the Control - A (with only high calorie diet, $n=10$ ) Experimental Group Grape Bark - B (high calorie diet + grape skin extract, $n=10)$ and experimental group Grape Seed - C (calorie diet + grape seed extract, $\mathrm{n}=10$ ). The results showed on average a reduction in serum total cholesterol by $14 \%$ and $17.65 \%$ in the control group, respectively, for the animals with diet based on bark and grape seed. There was no statistically significant difference in reducing cholesterol levels in the animals of both groups, so it is not possible to determine which grape component represents the best alternative in the prevention and control of hypercholesterolemia.
\end{abstract}

Key words: Fat Diet; Flavonoids; Hypercholesterolemia

\section{INTRODUÇÃO}


Hiperlipidemias são modificações no metabolismo em vivo onde há um aumento nos níveis de lipídeos na corrente sanguínea na corrente sanguínea, tal fator pode levar a aterosclerose que representa uma degeneração das grandes e médias artérias, se caracterizando pela presença de placas de ateromas no endotélio, todavia a aorta e os sítios coronarianos e cerebrais são os mais prevalentes (ARAÚJO, 2007).

Atualmente, está instituído que a hiperlipidemia importa um fator de risco significativo para o surgimento precoce de aterosclerose e complicações cardiovasculares (CASTRO et al., 2012).

Flavonóides são compostos polifenólicos biossintetizados a partir da via dos fenilpropanóides e do acetato. Estes compostos são universalmente encontrados em uma grande variedade de frutas, vegetais e algumas bebidas, incluindo chá e vinho, e por isso, são largamente consumidos na dieta humana (DORNAS et al., 2007).

Nesse sentido, sendo sabido que o aumento de LDL (Low-density lipoprotein) e especialmente LDL oxidadas são reconhecidas como fator de risco em Doença Arterial Coronariana, supõem-se que os flavonóides possam reduzir a geração ou aumento de radicais livres e atuar como antioxidante do LDL. Essa sugestão é apoiada por dados experimentais confirmados de que compostos fenólicos no vinho tinto inibem a oxidação de LDL (GIEHL 1 2007; SHIRAHIGUE, 2008; .LANDIM, 2009).

Assim, os flavonóides possuem atividade antioxidante na função protetora e no tratamento de doenças degenerativas mediadas por estresse oxidativo, onde evidências epidemiológicas crescentes sugerem que há correlação negativa entre consumo de alimentos ou bebidas ricas em polifenóis e a incidência de doenças cardiovasculares (LIMA, 2010; LOPES et al., 2000).

Assim, objetivou-se estudar o efeito redutor de colesterol do extrato de semente e de casca de uva (Vitis vinifera) em ratos Wistar submetidos a uma dieta hipercalórica in vitro.

\section{MATERIAL E MÉTODOS}

O estudo trata-se de uma pesquisa exploratória, experimental e comparativa, onde foram utilizadas ratas albinas primíparas da linhagem Wistar (250-300g) e suas descendências, compostas por 30 ratos machos, provenientes da colônia de criação do biotério da Faculdade de Ciências Médicas de Campina Grande(FCMCG). Os animais foram alojados em gaiolas de polipropileno com dimensão de 430x430x200 mm (CxLxA) num ambiente com temperatura de $23 \pm 1^{\circ} \mathrm{C}$, num ciclo de luz/escuridão $(12 \mathrm{~h})$ e tiveram livre acesso à água filtrada. Os animais foram acasalados na proporção de 01 macho para 03 fêmeas. O estado de prenhez foi determinado através da observação da presença de espermatozóides na secreção vaginal.

Os extratos de semente de uva e de casca de uva (Vitis vinifera) foram obtidos em uma loja de suplementos nutricionais orgânicos e naturais do município de Campina Grande (PB);

O extrato comercial de semente de uva (Vitis vinifera) foi utilizado em forma de pó, diluindo-se o conteúdo das cápsulas de $100 \mathrm{mg}$ em óleo, o extrato de casca de uva (Vitis vinifera) apresentava-se diretamente em forma de pó sendo pesadas em balança analíticas.

Foram formados três grupos: A - 10 (dez) Ratos Wistar machos que compuseram o Grupo Controle, com dieta de hipercalórica (ração padrão acrescida de 10\% de óleo de soja, cuja composição tem $57 \mathrm{~g} / 100 \mathrm{ml}$ de lipídios polinsaturados, $22 \mathrm{~g} / 100 \mathrm{ml}$ de gorduras monoinsaturadas e 13g/100ml de saturadas); B - 10 (dez) Ratos Wistar machos que compuseram o Grupo Experimental Casca, com dieta hipercalórica (ração padrão acrescida de $10 \%$ de óleo de soja e $100 \mathrm{mg} / \mathrm{kg} /$ dia de extrato de casca de uva); C - 10 (dez) Ratos Wistar machos que compuseram o Grupo Experimental Semente, com dieta hipercalórica (ração padrão acrescida de $10 \%$ de óleo de soja + $100 \mathrm{mg} / \mathrm{kg} / \mathrm{dia}$ de extrato de semente de uva).

A partir da $4^{\text {a }}$ semana de vida os animais foram submetidos a uma dieta hipercalórica para induzir ao desenvolvimento de aterosclerose e aumento dos níveis sanguíneos de colesterol.

Concomitantemente, cada grupo recebeu a sua dieta particular dessa forma o grupo B e C além da ração acrescida de óleo de soja, também consumiu $100 \mathrm{mg} / \mathrm{kg} / \mathrm{dia}$ de extrato de casca e semente de uva, respectivamente. A dieta teve a duração de 20 semanas, e nesse período foi feito o acompanhamento ponderal dos animais além da verificação dos níveis séricos de colesterol no início e no fim do experimento.

Em virtude da impossibilidade de administrar o extrato em pó da casca e semente de uva pela técnica de gavagem devido ao entupimento da agulha, foi feito um cálculo para diluir os extratos no óleo vegetal acrescido á ração.

Considerando que os ratos wistar consomem em média $30 \mathrm{~g}$ de ração por dia, para que consumissem $100 \mathrm{mg} / \mathrm{kg} / \mathrm{dia}$ de extrato de casca e semente de uva, respectivamente os grupos $\mathrm{B}$ e $\mathrm{C}$, os animais, separados em gaiolas em subgrupos de 04 , foram pesados a cada dois dias e calculado a quantidade de extrato que os animais de cada gaiola teriam que consumir conjuntamente nesse intervalo de 02 dias. Nesse sentido, misturou-se a quantidade de extrato para cada dois dias com a quantidade de ração consumida em média por os 04 animais de cada gaiola durante 02 dias, garantido a administração dos extratos na dosagem pretendida.

Foi realizado um estudo quantitativo e comparativo dos níveis sanguíneos de colesterol total em cada grupo, a fim de estabelecer o caráter benéfico redutor de colesterol de cada modalidade alimentar em estudo.

Nesse caso, os resultados obtidos nos ensaios foram expressos em média \pm SD (desvio-padrão), submetidos posteriormente ao teste de normalidade de shapiro-wilk e em seguida ao teste Tukey-Kramer. Além disso, os dados obtidos foram submetidos à análise estatística paramétrica, empregando-se testes de ANOVA One-Way. Foram considerados estatisticamente significativos os resultados que expressaram $p<0,05$.

Os procedimentos adotados no manejo dos animais seguiram as recomendações da Diretriz Brasileira de Prática Para o Cuidado e a Ultilização de Animais Para Fins Científicos e Didáticos - DBPA.

$\mathrm{O}$ projeto foi enviado à Comissão de Ética no Uso de Animal do Centro de Ensino Superior e Desenvolvimento (CEUA/CESED) para apreciação e seu 
início ocorreu após sua aprovação, com o protocolo de aprovação do projeto no CEUA/CESED nº 010/10022012.

\section{RESULTADOS E DISCUSSÃO}

Para fim de estudo quantitativo, o material dos grupos controle (A) e experimentos (B e C) foram analisados, apresentando os resultados na Tabela 1 e no Figura 1. A validade dos dados se confirma através dos testes estatísticos apontados na Tabela 2.

Tabela 1: Valores do colesterol total sérico dos animais do Grupo A, B e C após o experimento, em mg/dL.

\begin{tabular}{cccccc}
\hline \multicolumn{1}{c}{ Grupo A- Grupo Controle } & Grupo B - Extrato de Casca de Uva & Grupo C - Extrato de Semente de Uva \\
\hline A1 & 90,3 & B1 & 78,9 & C1 & 67,4 \\
A2 & 90,5 & B2 & 79,3 & C2 & 69,3 \\
A3 & 81,7 & B3 & 65,6 & C3 & 64,1 \\
A4 & 80,6 & B4 & 72,9 & C4 & 79,9 \\
A5 & 84,5 & B5 & 81,4 & C5 & 81,5 \\
A6 & 83,6 & B6 & 74,4 & C6 & 70,7 \\
A7 & 86,7 & B7 & 71,1 & C7 & 68,0 \\
A8 & 98,6 & B8 & 69,1 & C8 & 72,3 \\
A9 & 79,9 & B9 & 79,4 & C 9 & 67,9 \\
A10 & 85,2 & B10 & 68,9 & C10 & 68,4 \\
\hline
\end{tabular}

Figura 1. Médias dos níveis de Colesterol Total Sérico dos grupos A, B e C após o experimento, em mg/dL e Porcentagem de redução das Médias do Colesterol dos Grupos B e C em relação ao grupo A.

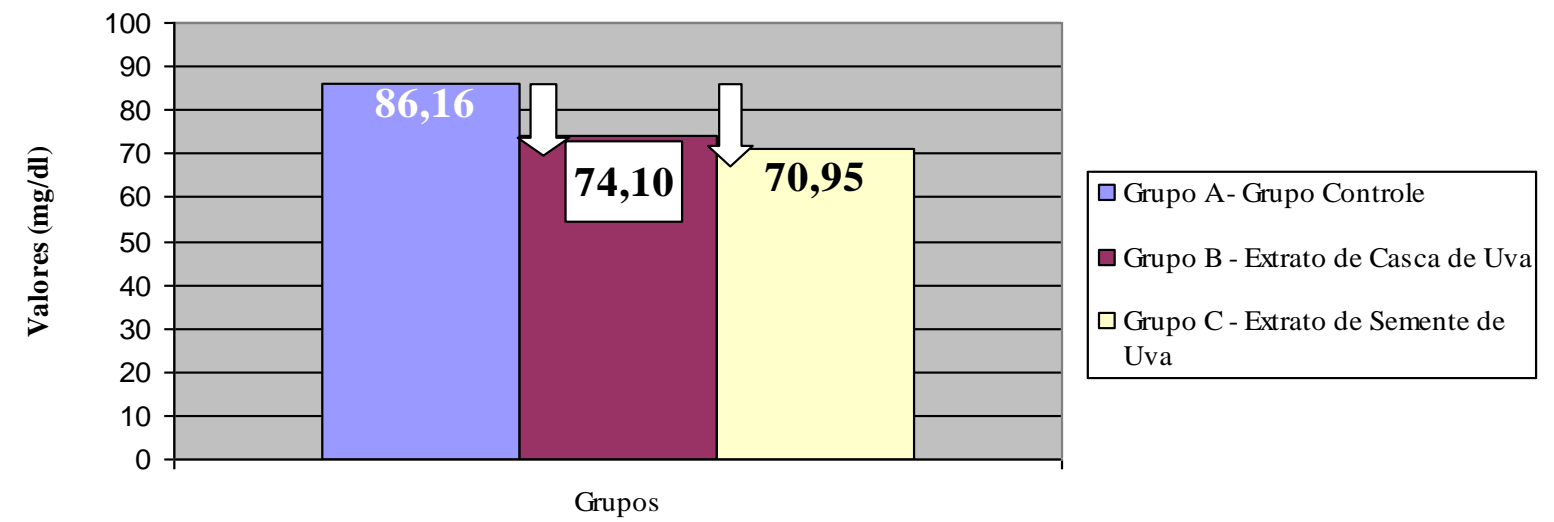

Tabela 2. Análise Estatística dos Níveis de Colesterol Total Sérico dos grupos A, B e C após o experimento.

\begin{tabular}{|c|c|c|c|c|c|}
\hline \multicolumn{6}{|c|}{ Testes Estatísticos } \\
\hline \multicolumn{2}{|c|}{ Grupo A- Grupo Controle } & \multicolumn{2}{|c|}{ Grupo B - Extrato de Casca de Uva } & \multicolumn{2}{|c|}{ Grupo C - Extrato de Semente de Uva } \\
\hline Média & 86,38 & Média & 74,10 & Média & 70,95 \\
\hline Desvio padrão & 5,83 & Desvio padrão & 5,44 & Desvio padrão & 5,58 \\
\hline Aumento sobre Grupo B & $16,28 \%$ & Redução sobre Grupo A & $14 \%$ & Redução sobre Grupo A & $17,65 \%$ \\
\hline Aumento sobre Grupo C & $21,44 \%$ & & & Redução sobre Grupo B & $4,25 \%$ \\
\hline Mediana & 84.8 & & 73,6 & & 68,8 \\
\hline Teste da Normalidade & Sim & & Sim & & Sim \\
\hline Distribuição Gaussiana & Sim & & Sim & & Sim \\
\hline $\begin{array}{l}\text { Teste paramétrico } \\
\text { (One Way ANOVA) }\end{array}$ & $\mathrm{p}=<0,0001$ & & $\mathrm{p}=<0,0001$ & & $\mathrm{p}=<0,0001$ \\
\hline Teste Tukey-Kramer & Sim & & Sim & & Sim \\
\hline Grupo A x Grupo B & $\begin{array}{l}q=6,847 / \\
p<0,001\end{array}$ & Grupo A x Grupo C & $\begin{array}{l}q=8,636 / \\
p<0,001\end{array}$ & Grupo B x Grupo C & $\begin{array}{c}q=1,788 / \\
p>0,05\end{array}$ \\
\hline
\end{tabular}

Os resultados indicaram que o Grupo $\mathrm{C}$ apresentou em média uma redução no valor de colesterol total sérico de 4,25\% em relação ao Grupo B (Tabela 2), porém não é possível assinalar uma diferença estatística entre a ação redutora de colesterol do extrato de semente de uva e do extrato de casca de uva (Vitis vinifera) para o tamanho da amostra e o desvio padrão, apesar da diferença pontual e dessa variação em torno de $3,22 \mathrm{mg} / \mathrm{dL}$ do aumento médio do colesterol total entre o os animais do Grupo B e os do Grupo C (Tabela 3), o que aponta para a necessidade de um estudo com uma amostra mais significativa.
Em contrapartida, houve redução estatisticamente significativa de ambos os grupos em relação ao grupo controle, com os valores de $14 \%$ de redução do colesterol do Grupo do Extrato de Casca de Uva e de 17,65\% do Grupo Extrato de Semente de Uva, comparando-os com o Grupo Controle (Figura 1), o que se pode confirmar pela média do aumento do colesterol entre o início e o fim do experimento, visto que houve um aumento de 26,14 $\mathrm{mg} / \mathrm{dL}$, para o Grupo A, e de apenas 14,21 mg/dL e 10,99 $\mathrm{mg} / \mathrm{dL}$, respectivamente para Grupo B e Grupo C, conforme Tabela 3 . 
Tabela 3: Médias do Colesterol Total Sérico dos animais do Grupo A, B e C, antes e após o experimento, em mg/dL.

\begin{tabular}{lccc}
\hline & $\begin{array}{c}\text { Grupo A- Grupo } \\
\text { Controle }\end{array}$ & $\begin{array}{c}\text { Grupo B - Extrato de Casca } \\
\text { de Uva }\end{array}$ & $\begin{array}{c}\text { Grupo C - Extrato de Semente } \\
\text { de Uva }\end{array}$ \\
\hline $\begin{array}{l}\text { Média no Início do } \\
\text { Experimento }\end{array}$ & $60,02( \pm 2,03)$ & $59,89( \pm 2,14)$ & $59,94( \pm 2,16)$ \\
Média no Fim do & $86,16( \pm 5,69)$ & $74,1( \pm 5,44)$ & $70.95(5,58)$ \\
Experimento & 26,14 & 14,21 & 10,99 \\
\hline Aumento do Colesterol & & & \\
\hline
\end{tabular}

As doenças cardiovasculares, incluindo doenças arteriais coronarianas, insuficiência cardíaca congestiva, acidente vascular cerebral, infarto agudo do miocárdio e hipertensão arterial sistêmica, constituem, atualmente, importantes patologias que acometem grande parte da população.

A importância de distúrbios de lipoproteínas plasmáticas e anormalidades no metabolismo lipídico caracterizadas por hiperlipidemia e/ou hipercolesterolemia como fator etiológico no desenvolvimento de doenças cardíacas coronarianas e aterosclerose potencial são, atualmente, cada vez mais apoiadas por considerável número de estudos populacionais e epidemiológicos (DORNAS et al., 2010).

Como é sabido, os flavonóides, presentes em frutas e vegetais e que fazem parte largamente e diariamente da nossa dieta, são compostos naturais polifenólicos de baixo peso molecular com atividade antioxidante sobre a LDL, ação antitrombótica e conseqüente atuação inibidora sobre a gênese da placa aterosclerótica (MOURA, 2006).

Nesse sentido, diversos estudos apresentaram a eficácia da uva, do suco de uva e do vinho tinto na prevenção da doença aterosclerótica, no controle da pressão arterial e do perfil lipídico, e uma revisão bibliográfica apontou que esse efeito está relacionado à propriedades antioxidadantes de polifenóis presentes na casca e sementes da uva (GIEHL et al., 2007).

Estudos isolados em animais concluíram que especificamente o extrato de casca de uva apresenta um efeito anti-hipertensivo, antioxidante e redutor dos níveis plasmáticos de colesterol e triglicerídeos (COSTA, 2008). Assim como, em outro estudo realizado com sementes de uva preta, investigadores revelaram uma recuperação do fluxo coronariano e do fluxo aórtico e melhora da pressão sanguínea em ratos reafirmando os seus efeitos cardioprotetores contra danos cardíacos da reperfusão (GIEHL et al., 2007).

Porém, apesar dessas pesquisas ressaltarem e reafirmarem $\mathrm{o}$ efeito antioxidante dos flavonóides presentes na semente e na casca de uva, não se delimitou dados concretos que possam servir como parâmetros para a determinação de uma dieta preferencial entre a casca e a semente de uva, com o melhor resultado terapêutico em relação à aterosclerose e a redução dos níveis sanguíneos do colesterol.

O presente trabalho objetivou estudar e comparar a ação hipocolesterolêmica dos flavonóides presentes na semente e na casca de uva (Vitis vinifera) através de seus estratos em ratos com uma dieta hipercalórica.

\section{CONCLUSÕES}

Ocorreu benefício para os níveis séricos de colesterol total de ambos os grupos com dieta a base de extrato de semente e de casca de uva em relação ao grupo sem dieta redutora, porém sem apresentar diferença estatística na comparação com o grupo controle.

\section{REFERÊNCIAS}

CASTRO, K. F.; ARTONI, S. M. B.; PACHECO, M. R. Perfil lipídico sérico de ratos tratados com surfactante. Braz. J. Vet. Res. Anim. Sci., Sao Paulo, v. 49, n. 3, p. 193-201, 2012.

COSTA, G. F. Efeito do extrato da casca de uva Vitis Vinífera (GSE) na pressão arterial, no perfil lipídico e glicídico e no estresse oxidativo em ratos espontaneamente hipertensos. Dissertação (Mestrado) Centro Biomédico da Universidade Estadual do Rio de Janeiro, Rio de Janeiro, 2008.

ARAUJO, A. L. Correlação entre dieta lipídica polinsaturada e aterogênese. Rev Angiol Cir Vasc., v. 5 n.5, p.15-22, 2007.

DORNAS, W. C., OLIVEIRA, T. T.; AUGUSTO, L. E. F.; NAGEM, T. J. Aterosclerose Experimental em Coelhos. Arq Bras Cardiol , v. 95, n.2, p. 272-278, 2010.

DORNAS, W. C.; OLIVEIRA, T. T.; RODRIGUESDAS-DORES, R. G.; SANTOS, A. F.; NAGEM, T. J. Flavonóides: potencial terapêutico no estresse oxidativo. Rev. Ciênc. Farm. Básica Apl., v. 28, n.3, p. 241- 249, 2007.

GIEHL, M. R.; DAL BOSCO, S. M.; LAFLOR, C. M.; WEBER, B. Eficácia dos flavonóides da uva,vinho tinto e suco de uva tinto na prevenção e no tratamento secundário da aterosclerose. Scientia Medica, Porto Alegre, v. 17, n. 3, p. 145-155, jul./set. 2007.

LANDIM, M. B. P. A hipercolesterolemia abole o efeito cardioprotetor do pré e pós-condicionamento isquêmico em um modelo experimental de isquemia e reperfusão em ratos. Tese (Doutorado) - Faculdade de Medicina da Faculdade de São Paulo, São Paulo, 2009.

LIMA, L. R. P. Efeito de flavonoides e de corantes do urucumsobre a hiperlipidemia induzida em coelhos. RBAC, vol. 42, n.1, p.69-74, 2010. 
LOPES, N. M.; OLIVEIRA. T. T.; NAGEM, T. J.;

PINTO, A. S. Farmacologia de flavonóides no controle hiperlipidêmico em animais experimentais. Biotecnologia, Ciência \& Desenvolvimento, v. 17, p. 18-22, 2000.

MOURA, M. D. Papel do flavonóide diocleína no desenvolvimento da aterosclerose em camundongos deficientes no gene que codifica a apolipoproteína E. Dissertação (Mestrado) - Faculdade de Farmácia da UFMG, Belo Horizonte, 2006.

SHIRAHIGUE, L. D. Caracterização química de extratos de semente e de casca de uva e seus efeitos antioxidantes sobre a carne de frango processada e armazenada sobre refrigeração. Dissertação (Mestrado) - Escola Superior de Agricultura Luis de Queiroz, Piracicaba, 2008. 